Artículo de Investigación

\title{
Evaluación del potencial geotérmico inverso y solar para reducir el consumo energético de una instalación hospitalaria en Panamá
}

\section{Evaluation of reverse geothermal and solar potential to reduce the energy consumption of a hospital facility in Panama}

\author{
María Him Moya 1(i), Angienely Humphries ${ }^{1(D)}$, Pedro Fuentes ${ }^{1(D)}$, Miguel Chen Austin 1,2, *(D) \\ ${ }^{1}$ Facultad de Ingeniería Mecánica, Universidad Tecnológica de Panamá, Ciudad de Panamá, Panamá; 0819-07289; \\ maria.him1@utp.ac.pa; angienely.humphries@utp.ac.pa; pedro.fuentes1@utp.ac.pa \\ ${ }^{2}$ Centro de Estudios Multidisciplinarios en Ciencias, Ingeniería y Tecnología (CEMCIT-AIP), Ciudad de Panamá, Panamá; 0819-
} 07289

Citación: Him, M., Humphries, A., Fuentes, P., \& Chen, M., (2022). Evaluación del potencial geotérmico inverso y solar para reducir el gasto energético de una instalación hospitalaria en Panamá. Novasinergia. 5(1). 83-99. https://doi.org/10.37135/ns.01.09. 06

Recibido: 25 septiembre 2021

Aceptado: 29 enero 2022

Publicado: 31 enero 2022

Novasinergia

ISSN: 2631-2654
*Correspondencia: miguel.chen@utp.ac.pa

Resumen: Uno de los principales problemas que afecta al medio ambiente es el alto consumo de fuentes fósiles el cual se ha buscado mitigar a través de la utilización de fuentes renovables de energía. Entre los grandes consumidores energéticos se encuentran las edificaciones hospitalarias dado su funcionamiento de manera ininterrumpida. Esta investigación busca reducir el consumo energético de una instalación hospitalaria en la ciudad de Panamá. mediante la evaluación del potencial geotérmico inverso y solar de la zona. Para lograr este objetivo se modeló la edificación usando el software DesignBuilder, se dimensionó un intercambiador de calor basado en la simulación realizada, se analizó de forma teórica la temperatura del suelo para conocer los días de operatividad del sistema geotérmico y se calculó el número de paneles fotovoltaicos a utilizar. Se promediaron ahorros de $47.83 \%$ en gastos por refrigeración lo que llevaría a una reducción de $9.29 \%$ de la demanda eléctrica mensual, concluyendo que es un porcentaje significativo de ahorro para este tipo de edificaciones de comprobarse y aplicarse de manera práctica las hipótesis presentadas. Además, sienta las bases para llevar las edificaciones hospitalarias a un consumo de energía casi nulo.

Palabras clave: Ahorro energético, consumo energético, energía geotérmica, hospital, paneles fotovoltaicos.

Abstract: One of the main problems affecting the environment is the high consumption of fossil fuels, which has been mitigated by using renewable energy sources. Hospital buildings stand among the major energy consumers due to their uninterrupted operation. This research seeks to reduce the energy consumption of a hospital facility in Panama City by evaluating the inverse geothermal and solar potential of the area. To achieve this objective, the building was modeled using DesignBuilder software, a heat exchanger was sized based on the simulation, the ground temperature was analyzed theoretically to determine the days of operation of the geothermal system, and the number of photovoltaic panels to be used was calculated. Savings of $47.83 \%$ in cooling costs were averaged, which would lead to a reduction of $9.29 \%$ of the monthly electricity demand, concluding that this is a significant percentage of savings for this type of building if the hypotheses presented are verified and applied in a practical manner. It also lays the groundwork for bringing hospital buildings to near-zero energy consumption.

Keywords: Energy consumption, energy savings, geothermal energy, hospital, photovoltaic panels. 


\section{Introducción}

Se pronostica para el año 2030 un alza de 57\% en el consumo de energía primaria mundial de seguir la tendencia actual de aumento en el consumo eléctrico y en el costo del petróleo y gas natural, lo que presenta la necesidad de reducir el consumo energético, destacando el sector hospitalario entre los tipos de edificaciones con mayor consumo dado su funcionamiento continuo los 365 días del año, 24 horas al día. Es por ello por lo que la integración de nuevas fuentes energéticas en los hospitales para reducir su gasto eléctrico, de calefacción y refrigeración tiene alta importancia, destacando el caso de Panamá que, a la fecha de esta investigación, no presenta reportes en el área mencionada.

Entre estas nuevas fuentes de energía, el estudio se enfocará en el uso de la energía geotérmica en combinación con la energía solar fotovoltaica. La energía geotérmica es "una energía limpia y renovable que aprovecha el calor del subsuelo para climatizar y obtener agua caliente sanitaria de forma ecológica". Esta fuente también se puede aprovechar para la climatización de edificaciones donde esta "cede o extrae calor de la tierra, según se requiera obtener refrigeración o calefacción, a través de un conjunto de colectores enterrados en el subsuelo por las que circula una solución líquida" (Tokman, 2008). Poniendo a Panamá en el contexto de su potencial geotérmico, el reporte "Geotermia en Panamá: un análisis de necesidades", menciona que primeramente en el país existe una gran necesidad de nuevas evaluaciones técnicas para medir de forma concluyente el potencial para la energía geotérmica en el istmo, siendo la más reciente estimación del año 2006, realizada por West Japan Engineering Consultants, mostrando como resultados $24 \mathrm{MW}$ posibles en el área de Barú - Cerro Colorado y $18 \mathrm{MW}$ en el área del Valle de Antón, con $100{ }^{\circ} \mathrm{C}$ a $140{ }^{\circ} \mathrm{C}$ en profundidades de 600 a $1000 \mathrm{~m}$ (ETESA, 2010). Por lo que se observa la posibilidad de la implementación de energía geotérmica, clasificada "de media entalpía" que permitiría aplicaciones como la generación de electricidad y el aprovechamiento del calor de forma directa en sistemas de calefacción industriales o urbanos (Grupo Visiona, 2013). Todo esto apunta a la posibilidad del desarrollo de edificaciones sostenibles de bajo consumo en el país.

Por otro lado, la energía solar fotovoltaica se define como "la transformación directa de la radiación solar en electricidad a través de los paneles fotovoltaicos. En estos, la radiación solar excita los electrones de un dispositivo semiconductor generando una pequeña diferencia de potencial"(Grupo Ibal, 2019). A su vez el sistema fotovoltaico descrito en este estudio estará constituido de paneles fotovoltaicos de materiales de alta eficiencia, como es el caso más común la utilización de silicio monocristalino, este material suele ser más caro que las celdas policristalinas, pero permite un mayor rendimiento debido a la alta sensibilidad y la captación solar, incluso en climas adversos, niebla o tormenta y cuando se busque aumentar la producción y aprovechar el espacio de utilidad para su colocación (Elvis, Soto, \& Dixon, 2005). A pesar de que hasta el momento la energía solar fotovoltaica ocupe el $2.15 \%$ dentro de la matriz energética panameña, es un sistema de producción de energía limpia que va en aumento (ETESA, 2018) ya que la radiación solar promedio por día en Panamá es de $4.8 \mathrm{kWh} / \mathrm{m}^{2}$, siendo la región más favorecida el sur de Chiriquí donde el promedio por día está situado en los $5.4 \mathrm{kWh} / \mathrm{m}^{2}$ (Secretaría Nacional de Energía, 2015). Según datos de (Autoridad Nacional de los Servicios Públicos, 2018) Panamá cuenta con 241 
instalaciones de generación solar de las cuales, la provincia de Panamá Oeste ocupa el primer lugar en el interior del país con $23 \%$, seguida de Coclé con $10 \%$, Chiriquí con $7 \%$, Veraguas con $5 \%$, Colón con $4 \%$, Herrera con $2 \%$ y Los Santos con $2 \%$, mientras que la provincia de Panamá, a nivel nacional, concentra la mayor cantidad de sistemas fotovoltaicos de autoconsumo instalados con un $47 \%$. Adicionalmente se han realizado estudios en instalaciones hospitalarias a nivel internacional donde se destacan que al instalar sistemas solares fotovoltaicos se evita la emisión al medioambiente de 44 toneladas de gases de $\mathrm{CO}_{2}$, la institución hospitalaria estima un ahorro anual de 8376 dólares gracias al 18.8\% de ahorro energético producido (Jiménez, Leal, \& Garzón, 2016).

Las medidas mencionadas en conjunto a otros elementos pueden contribuir con otras estrategias como lo son las edificaciones a energía neta cero (NZEB por sus siglas en inglés), las cuales se entienden como: un edificio que tiene un rendimiento energético muy alto, la casi nula o muy baja cantidad de energía requerida debe ser cubierta en una medida muy significativa por energía de fuentes renovables, incluyendo la energía de fuentes renovables producida en el lugar o en las cercanías. Esto no quiere decir que la edificación no consuma energía, sino que esta consume prácticamente lo mismo que produce (Teba, 2020). Estas edificaciones serían un eslabón más en la cadena que lleve a Panamá a una reducción considerable de sus emisiones de gases invernadero, a la vez de contribuir al mejoramiento de los servicios de la red eléctrica. Para llegar a esto se puede iniciar incidiendo directamente sobre las fuentes de consumo energético, por ende, muchas empresas aplican a la certificación Liderazgo en Energía y Diseño Ambiental (LEED por sus siglas en inglés) para alcanzar este objetivo, así se presenta en un estudio cuya finalidad radica en la promoción de la salud pública direccionándola hacia la sostenibilidad, seguridad y sistemas de control, además de los pasos seguidos para la obtención de la certificación, obteniendo como resultado la categoría plata de la certificación para un hospital de $106000 \mathrm{~m}^{2}$, con capacidad para 200 camas, cumpliendo con los criterios establecidos en la certificación: sostenibilidad del sitio, eficiencia en el manejo del agua, energía y atmósfera, materiales y recursos, calidad del ambiente interior e innovación (Uribe \& Arboleda, 2015). También, es destacable el caso de una tesis realizada en España donde se buscó una alternativa renovable de abastecimiento energético en un hospital, tomando en consideración el clima de la zona y los recursos con los que se cuentan en esa área, evaluando así la energía solar térmica, biomasa, geotermia y mini eólica, resultando como más factible el abastecimiento de caldera por biomasa forestal, ya que se cubre más de un $95 \%$ de la demanda en energía térmica de la instalación (Valdés, 2018). Igualmente se presenta el caso de un hospital en Massachusetts, uno de los hospitales más importantes de Estados Unidos, ubicado específicamente en Boston con una extensión $30905.50 \mathrm{~m}^{2}$, en donde su gasto de energía más significativo se da en el sistema de climatización. Como resultado se obtuvo que, de aplicarse la estrategia propuesta, las instalaciones del MGH (Massachusetts General Hospital) podrían ahorrar hasta un 60\% de los costes de climatización al año, lo que representaría un ahorro de 1218626 dólares al año.

Como otro ejemplo sobre las medidas de ahorro en edificaciones hospitalarias, en un estudio se muestra que con la implementación de medidas activas y pasivas de ahorro energético en edificaciones de menos de $5000 \mathrm{~m}^{2}$ se pueden obtener ahorros de hasta $8.60 \mathrm{kWh} / \mathrm{m}^{2}$ por 
año y $6.88 \mathrm{kWh} / \mathrm{m}^{2}$ en construcciones de más de $5000 \mathrm{~m}^{2}$, representado ahorros en costos de 6192.00 y 37152.00 euros al año, respectivamente (Zyvith et al., 2018).

La implementación de este tipo de acciones ha llevado al estudio de la viabilidad de construcción de edificios NZEB, específicamente en las ciudades de Guatemala, Costa Rica, Cuba, El Salvador, Puerto Rico, Nicaragua, Panamá, Belice, San Pedro Sula y Acajutla, aplicando el prototipo experimental construido en la Universidad Centroamericana José Simeón Cañas en El Salvador para encontrar el balance óptimo de energía neta cero anual en estas ubicaciones mediante simulaciones energéticas. Esto obtuvo como resultado entre 10 a 28 modelos optimizados que indicaron que si la edificación prototipo se colocara en estas ciudades se necesitarían entre 25 a 27 paneles solares para poder ser considerados como NZEB (Hernández, Lemus, Solano, \& Martínez, 2020).

Conociendo entonces el alto consumo de energía generado por las instalaciones hospitalarias y las distintas formas en las que se puede disminuir, el objetivo principal de esta investigación es evaluar la aplicación de un mecanismo que pueda reducir el consumo por refrigeración de la instalación mediante un sistema geotérmico inverso del tipo airetierra. Adicional a esto, se implementarán paneles fotovoltaicos para contribuir al ahorro energético. Estos métodos en conjunto, a mediano o largo plazo, pueden aportar al mejoramiento del sistema hospitalario, además de complementar y/o brindar información relevante sobre técnicas de ahorro energético en el país y sentar las bases para llevar las edificaciones hospitalarias a ser NZEB.

\section{Metodología}

En la figura 1 se observa la ruta de metodología que se siguió para el desarrollo del estudio.

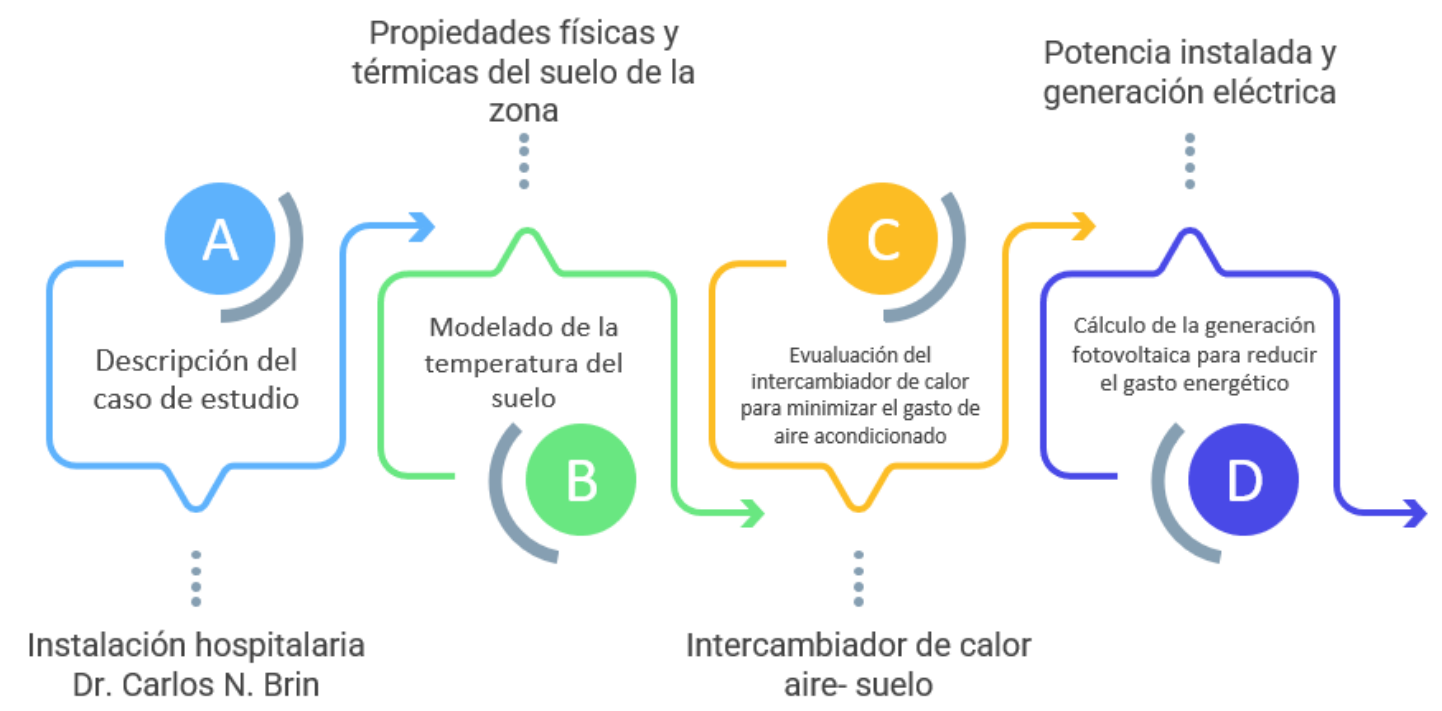

Figura 1: Diagrama metodológico del estudio presentado.

\subsection{Descripción del caso de estudio}

El caso de estudio corresponde a la policlínica Dr. Carlos N. Brin ubicada en el corregimiento de San Francisco, Distrito de Panamá, adyacente al Parque Recreativo Omar 
Torrijos, con coordenadas específicas $8^{\circ} 59^{\prime} 45^{\prime \prime} \mathrm{N} 79^{\circ} 30^{\prime} 50^{\prime \prime} \mathrm{W}$, orientado desde su entrada a $45^{\circ}$ respecto al norte, mostrado en la figura 2.

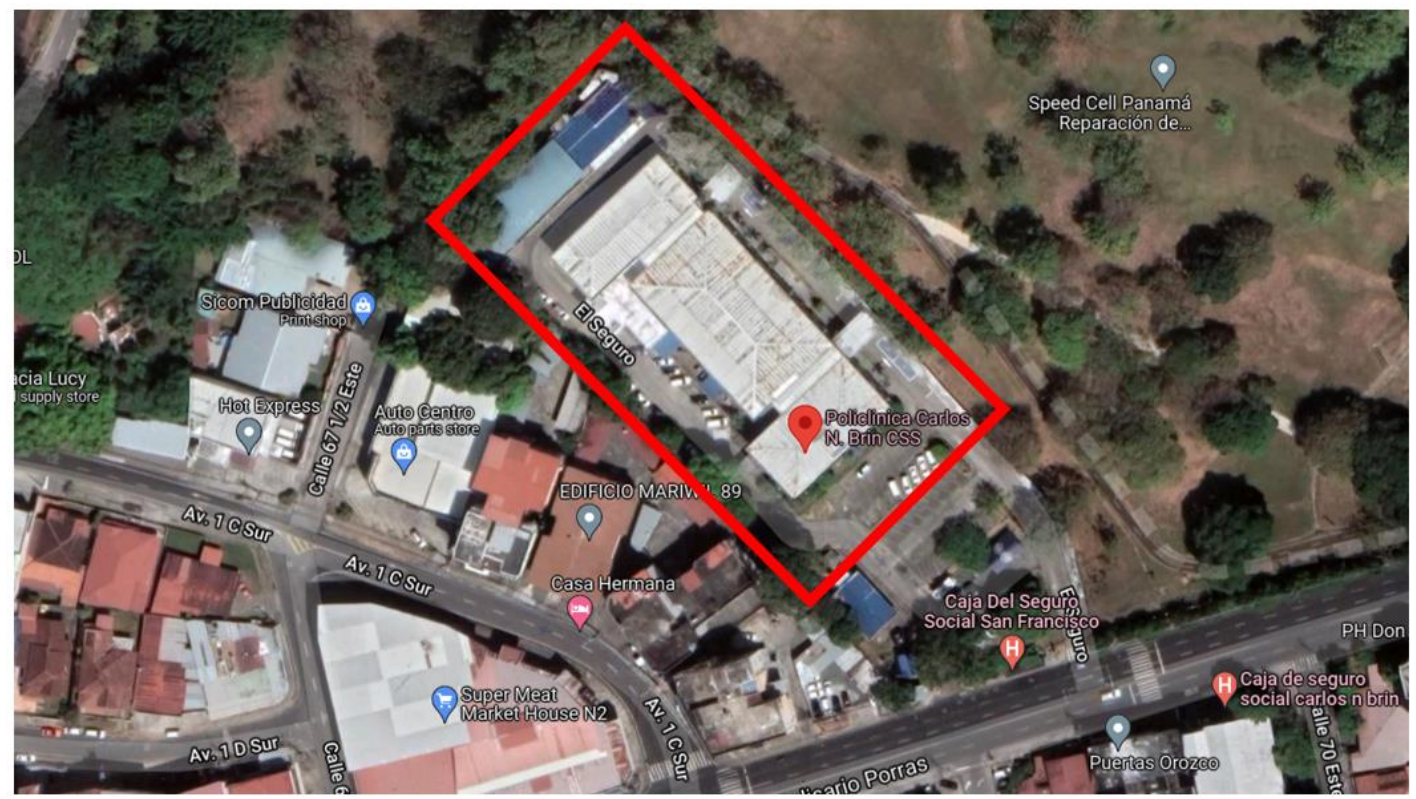

Figura 2: Mapeo y áreas aledañas a la edificación hospitalaria del caso de estudio (Google Earth, 2021). Se aprecia el área abierta en la parte trasera con árboles, la cual beneficia a la edificación, y el área delantera totalmente pavimentada.

Obtenidos los planos originales en la Dirección Nacional de Infraestructura y Servicios de Apoyo (DINISA), de la Caja de Seguro Social (CSS), se realizó un modelo de la estructura hospitalaria en el software DesignBuilder, una plataforma especializada en simulaciones energéticas de edificios, fabricada por la compañía británica Stroud, de la cual se empleó en esta investigación la versión 6. La instalación cuenta con dos plantas con una superficie total de $4622.56 \mathrm{~m}^{2}$ como se describe en la tabla 1 y una altura de $9 \mathrm{~m}$, apoyada sobre una superficie de concreto. Se observa una vista general de la edificación en la figura 3.

Tabla 1: Superficies de planta de la edificación.

Superficies por planta $\left(\mathrm{m}^{2}\right)$

$\begin{array}{cc}\text { Planta baja } & 2285.63 \\ \text { Planta alta } & 2336.93 \\ \text { Total } & 4622.56\end{array}$

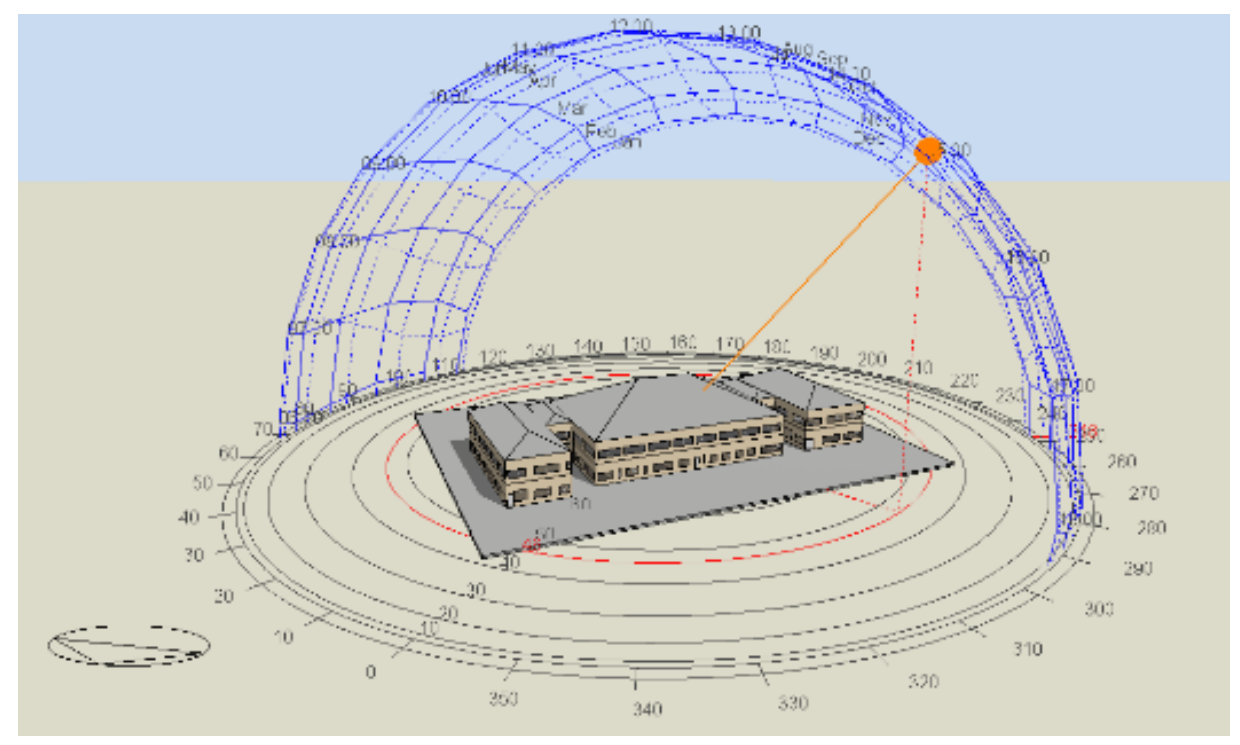

Figura 3: Vista general de la edificación seleccionada para estudio modelada en el software DesignBuilder. 
Dado que no se presentan datos oficiales del consumo eléctrico y térmico de las áreas de la policlínica, se realizó una estimación mediante herramientas bibliográficas como se muestra en la tabla 2, una vez definido esto se procede a realizar la simulación.

Tabla 2: Consumo eléctrico en la edificación hospitalaria correspondiente a luminarias y equipos.

\begin{tabular}{ccc}
\hline Planta & $\begin{array}{c}\text { Luminarias } \\
\left(\mathrm{W} / \mathrm{m}^{2}\right)\end{array}$ & $\begin{array}{c}\text { Equipos y otros } \\
\left(\mathrm{W} / \mathrm{m}^{2}\right)\end{array}$ \\
\hline Planta inferior & 4.5 & 680993.0 \\
Planta superior & 3.7 & 239871.6 \\
\hline
\end{tabular}

\subsection{Modelado de la temperatura del suelo}

Para conocer las propiedades del suelo en Panamá, para el cual se ha establecido como tipo general una combinación de arenisca y caliza, los informes (Autoridad Nacional de Ambiente, 2011; Escalante \& Villalba, 2018) detallan los tipos y propiedades térmicas de este como lo muestra la tabla 3.

Tabla 3: Propiedades físicas y térmicas generales del suelo en Panamá.

\begin{tabular}{cccc} 
Propiedad & Símbolo & Valor & Unidad \\
\hline Conductividad térmica & $\lambda$ & 4.7 & $\mathrm{~W} / \mathrm{mK}$ \\
Capacidad térmica volumétrica & $\varrho^{-C_{P}}$ & 2.80 & $\mathrm{MJ} / \mathrm{m}^{3} \mathrm{~K}$
\end{tabular}

Siguiendo lo planteado en la investigación (Belatrache, Bentouba, \& Bourouis, 2017), la ecuación (1) permite el cálculo de la difusividad térmica, la cual a su vez se emplea en el en el cálculo de la temperatura del suelo, realizado mediante la ecuación (2).

$$
\begin{gathered}
\alpha=\frac{\lambda}{\varrho C_{P}} \\
T(z, t)=T_{\text {sup }}+\mathrm{A}_{s}\left(\mathrm{e}^{-\mathrm{z}\left(\sqrt{\frac{\pi}{365 \alpha}}\right)} \cos \left[\frac{2 \pi}{365}\left(\mathrm{t}-\mathrm{t}_{0}\right)-\frac{\mathrm{z}}{2} \sqrt{\frac{365}{\pi \alpha}}\right]\right)
\end{gathered}
$$

En la que $T_{\text {sup }}$ es la temperatura anual promedio en ${ }^{\circ} \mathrm{C}, A s$ la amplitud de la variación de la temperatura superficial de suelo en ${ }^{\circ} \mathrm{C}, z$ representa la profundidad desde la superficie del suelo en $\mathrm{m}, t$ corresponde al día al año analizado y $t_{0}$ al día más caliente del año. Dado que As es un criterio puramente experimental y los datos sobre este no se encuentran en la literatura, para los propósitos de esta investigación se varía el valor según el caso que se analiza. Obteniendo los valores de $T_{\text {sup }}, t$ y to de la simulación realizada y variando los valores de $z$ y As se conocen entonces las diversas temperaturas que teóricamente el suelo sería capaz de proporcionarle al sistema geotérmico.

\subsection{Evaluación del intercambiador de calor aire - suelo}

Se escogió el diseño de un intercambiador de calor aire - suelo, como se muestra en la figura 4, por la simplicidad del sistema al ser este sin retorno y los requerimientos energéticos en su funcionamiento son prácticamente nulos. 


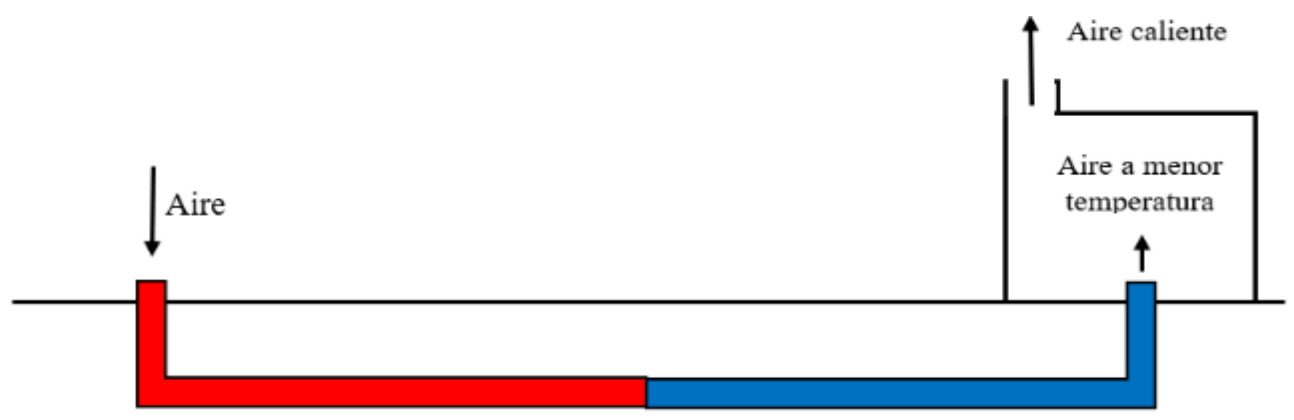

Figura 4: Esquemático del intercambiador de calor aire - tierra para sistema de climatización del hospital.

Se consideran dos casos de estudio: para el caso uno, se calcula la longitud total de la tubería del sistema según las ecuaciones de convección forzada interna asumiendo un valor en la temperatura interior del recinto de $25.5^{\circ} \mathrm{C}$ y en el suelo de $25^{\circ} \mathrm{C}$ (Cengel, Yunus A.; Ghajar, 2011). Se asumió que la transferencia de calor entre la tubería y el suelo se da de manera ideal, calculando el flujo másico, simbolizado como min, según la ecuación (3).

$$
\dot{\mathrm{m}}=\frac{\text { achVol}_{1} \mathrm{Q}}{3600}
$$

Para obtener el coeficiente de transferencia de calor (h) como se muestra en la ecuación (4), se requiere el cálculo del número Reynolds (Re) con la ecuación (5) y el número local de Nusselt $(\mathrm{Nu})$, este último se puede obtener de la conocida ecuación de Dittus-Boelter, mostrada en la ecuación (6). Para el número de Prandtl se mantiene un valor fijo de 0.7 para el aire y en cuanto al número de Reynolds se establece un rango descrito en: flujos internos $\operatorname{Re}<2000$, flujo laminar si $4<\operatorname{Re}<2000$, zona de transición para valores entre $2000<\operatorname{Re}<$ 4000 y flujo turbulento si $\operatorname{Re}>4000$. Se conoce también que el flujo laminar es un flujo generalmente experimental.

$$
\begin{gathered}
\mathrm{h}=\frac{\mathrm{Nud}}{\mathrm{k}} \\
\mathrm{Re}=\frac{\mathrm{\rho vd}}{\mu} \\
\mathrm{Nu}=0.023 \operatorname{Re}^{0.8} \operatorname{Pr}^{\frac{1}{3}}
\end{gathered}
$$

La longitud horizontal total requerida se calcula según la ecuación (7), la cual incorpora el cálculo del área superficial de transferencia de calor.

$$
\mathrm{L}=-\frac{\dot{m} c p}{\pi \mathrm{dh}}\left(\ln \left|\frac{\mathrm{T}_{\mathrm{e}}-\mathrm{T}_{\mathrm{S}}}{\mathrm{T}_{\mathrm{i}}-\mathrm{T}_{\mathrm{s}}}\right|\right)
$$

Dentro de estas ecuaciones se reemplazan los datos mostrados en la tabla 4.

Tabla 4: Datos considerados para el cálculo del intercambiador de calor aire-tierra

\begin{tabular}{cccc}
\hline Datos & Símbolo & Valor & Unidad \\
\hline Tasa de renovamiento del aire interior & $\mathrm{ach}$ & 0.5238 & $1 / \mathrm{h}$ \\
Calor específico & $\mathrm{cp}$ & 1007 & $\mathrm{~J} / \mathrm{kgK}$ \\
Temperatura de salida & $\mathrm{Te}$ & 25.5 & ${ }^{\circ} \mathrm{C}$ \\
Temperatura de entrada & $\mathrm{Ti}$ & 27.2 & ${ }^{\circ} \mathrm{C}$ \\
Volumen de planta baja & $\mathrm{Voll}$ & 10285.3 & $\mathrm{~m}^{3}$ \\
Densidad & $\mathrm{Q}$ & 1.184 & $\mathrm{~kg}^{3} \mathrm{~m}^{3}$ \\
Diámetro del tubo & $\mathrm{d}$ & 0.08 & $\mathrm{~m}$ \\
Temperatura del suelo & $\mathrm{Ts}$ & 25 & ${ }^{\circ} \mathrm{C}$ \\
Conductividad térmica & $\mathrm{k}$ & 0.02 & $\mathrm{~W} / \mathrm{mK}$ \\
\hline
\end{tabular}


Continuación tabla 4: Datos considerados para el cálculo del intercambiador de calor aire-tierra

\begin{tabular}{cccc}
\hline Datos & Símbolo & Valor & Unidad \\
\hline Viscosidad dinámica & $\mu$ & $1.86 \times 10^{-5}$ & $\mathrm{~kg} / \mathrm{ms}$ \\
Número de Prandtl & $\operatorname{Pr}$ & 0.7 & - \\
Velocidad promedio del aire & $\mathrm{v}$ & 2 & $\mathrm{~m} / \mathrm{s}$ \\
\hline
\end{tabular}

una vez obtenida la longitud de tubería como se muestra en el caso 1, en el caso 2, se varía el valor de As y por tanto, el valor de la temperatura interna del recinto, con lo que se conocerá las temperaturas de confort que el sistema podría proveer, tomando en cuenta que el confort térmico es la condición en la que se expresa satisfacción con el ambiente térmico y que este depende de características del recinto como la temperatura, humedad relativa, presión de vapor del aire, velocidad del aire y las características de los ocupantes (Secretaría Nacional de Energía, 2016), por lo que su evaluación es subjetiva y para esta investigación se toman en cuenta los puntos presentados en la tabla 4.

\subsection{Cálculo de la generación fotovoltaica}

La instalación hospitalaria en estudio cuenta con tres secciones de techo de cuatro aguas, que en conjunto tienen un área total de $1848.33 \mathrm{~m}^{2}$ tomando en cuenta la inclinación.

Para conocer la electricidad generada por los paneles se parte de la ecuación (8) que permite obtener la potencia instalada de los paneles y aplicar este dato en la ecuación (9), referente a la electricidad generada por día.

$$
\begin{gathered}
\mathrm{P}_{\text {ins }}=\mathrm{NP}_{\mathrm{n}} \\
\mathrm{W}_{\text {el }}=\frac{\phi_{\text {daily }}}{\phi_{\text {STC }}} \mathrm{k}_{\text {eff }} \mathrm{P}_{\text {ins }}
\end{gathered}
$$

En donde $\mathrm{P}_{\mathrm{ins}}$ es la potencia instalada en $\mathrm{kW}, \mathrm{N}$ es el número de paneles fotovoltaicos y $\mathrm{P}_{\mathrm{n}}$ es la potencia nominal del panel. $W_{\text {el }}$ es la electricidad generada por día en $\mathrm{kWh}$, $\phi_{\text {daily }}$ es la irradiación solar diaria sobre el módulo en $\mathrm{kWh} / \mathrm{m}^{2}, \phi_{\text {STC }}$ es la irradiación solar para condiciones de ensayo estándar $\left(1 \mathrm{~kW} / \mathrm{m}^{2}\right)$ y $\mathrm{k}_{\mathrm{eff}}$ es el factor de corrección del rendimiento del sistema, que según (Zomer, Urbanetz, \& Rüther, 2011) se recomienda que sea 0.85.

\section{Resultados}

\section{Consumo total del edificio}

Los resultados obtenidos luego de realizada la simulación en el software DesignBuilder se detallan en las figuras a continuación. En la figura 5 se observa el consumo energético hospitalario por categorías en equipos de consumo como equipos hospitalarios, iluminación y aire acondicionado. Los resultados posicionan a los equipos médicos con el mayor gasto, debido a la alta demanda que tienen estos en los rangos de operatividad en las horas ocupadas de la instalación. 


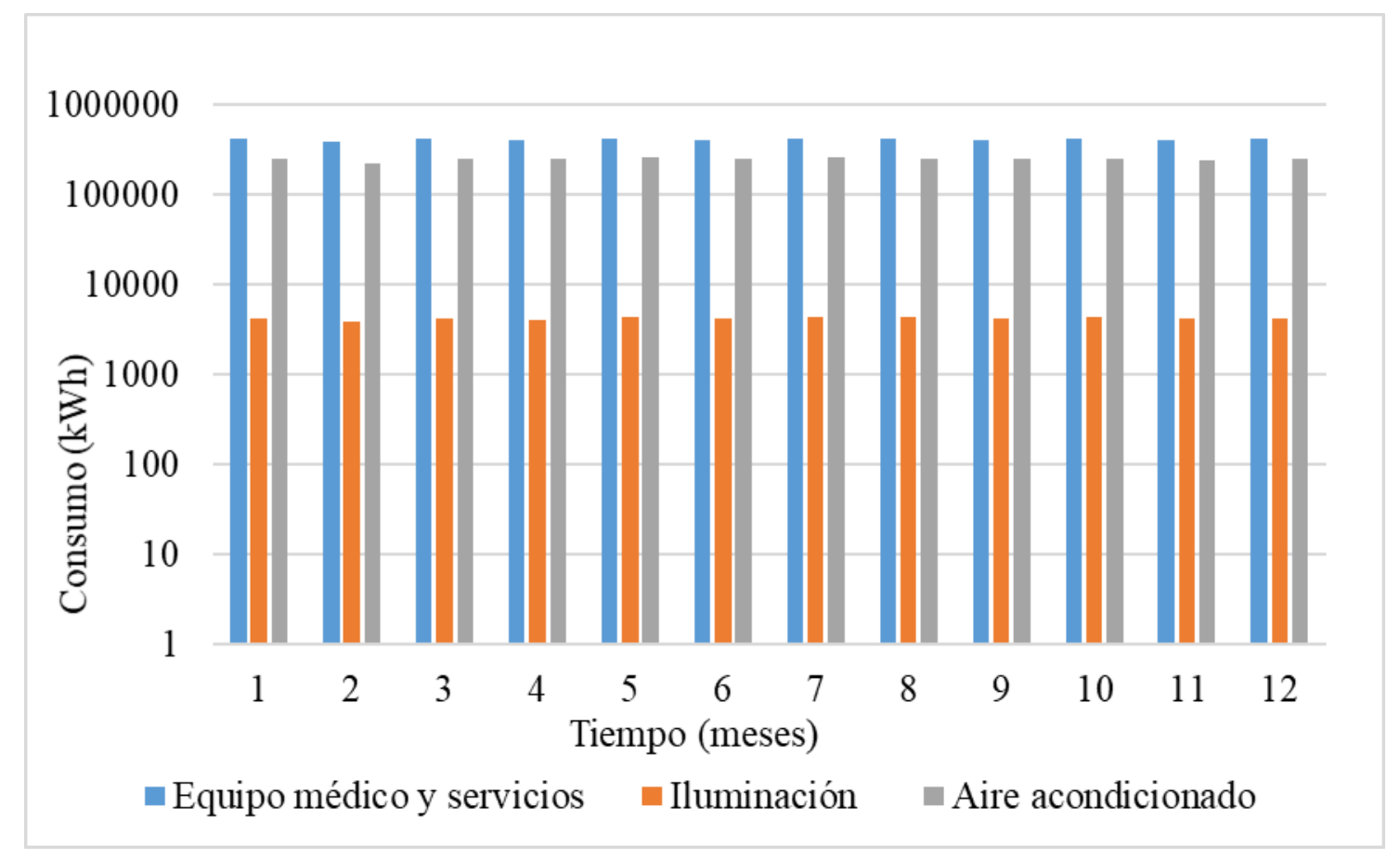

Figura 5: Consumo eléctrico hospitalario anual, basado en calendario enero - diciembre.

En la figura 6 se observa la tasa de calor mensual para las distintas superficies de la edificación desde el punto de vista del aire interior de este. Entre las pérdidas de calor para valores negativos y las ganancias para positivos, a través de las diferentes superficies de la estructura, se destacan los vidrios y paredes tomando en consideración las diferentes fuentes de calor presentadas en la figura 7, en las distintas áreas a través del tiempo con datos tomados de la simulación en DesignBuilder.

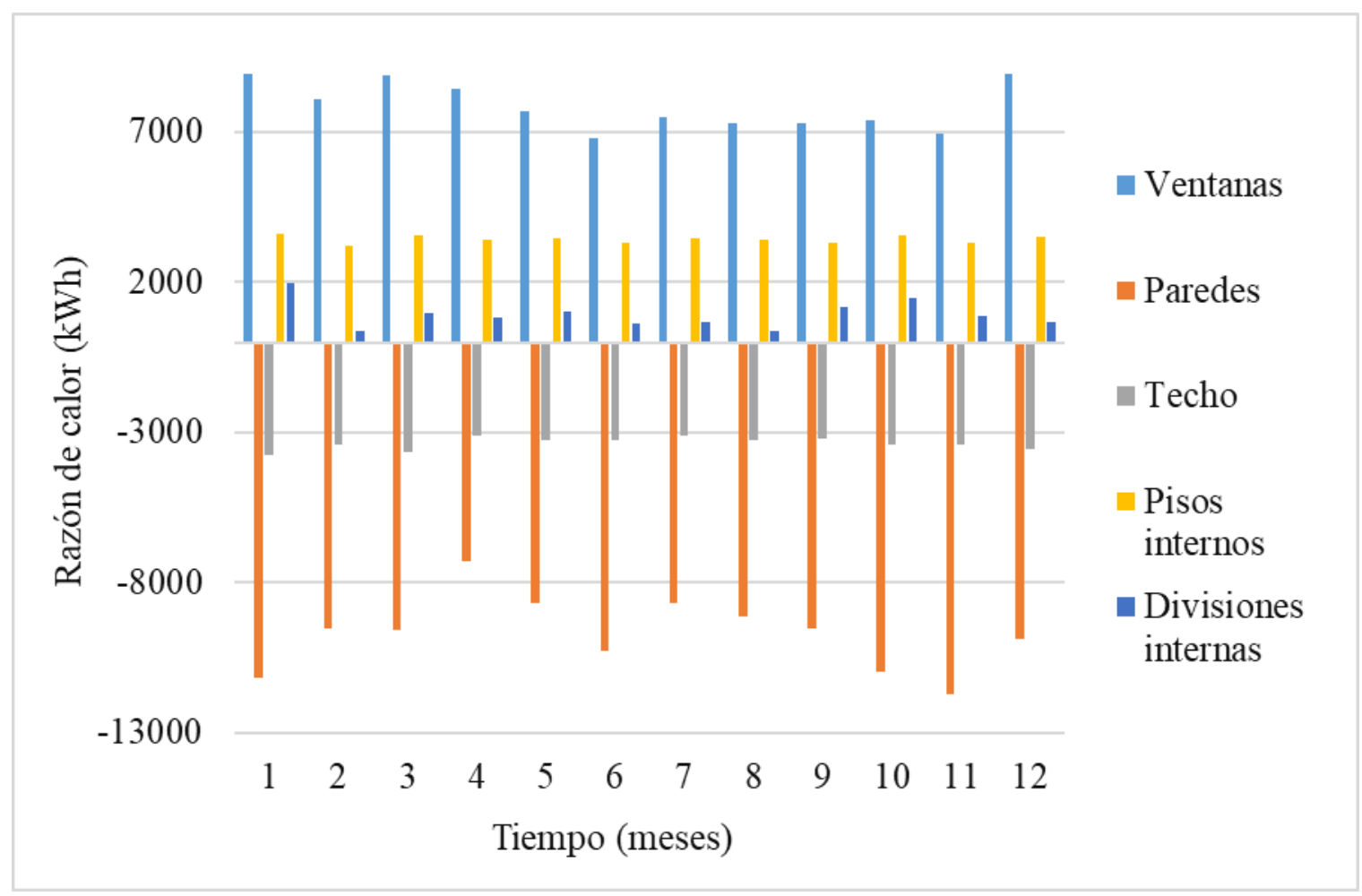

Figura 6: Tasa de calor mensual, partiendo del mes de enero. 
El mayor consumo de energía térmica para las unidades mencionadas en la figura 7 , son los equipos médicos, seguido de los equipos de oficina, pero bastante inferiores a los ya mencionados, desde el punto de vista de aire interior del recinto.

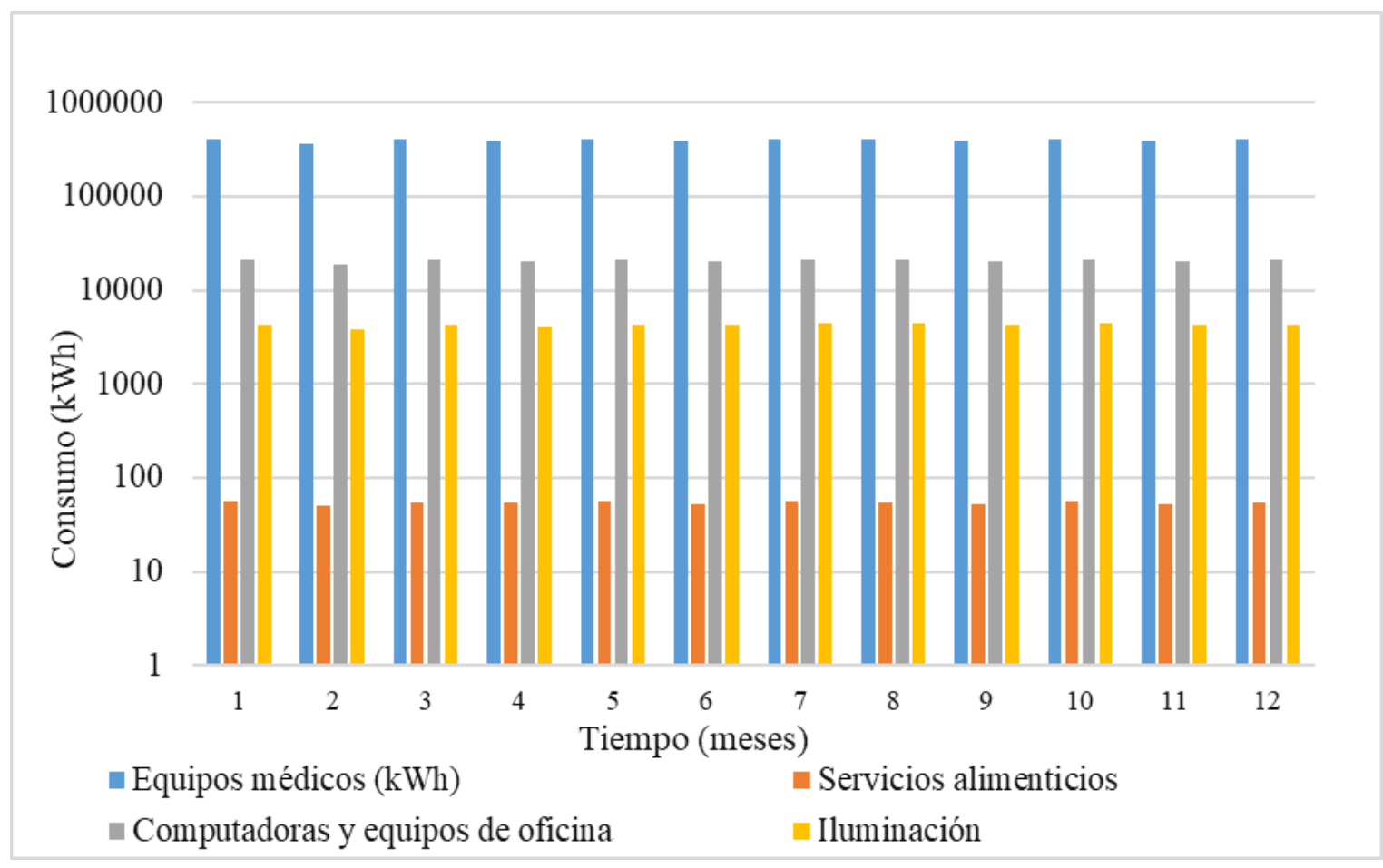

Figura 7: Consumo de energía térmica por categoría de equipo, partiendo del mes de enero.

La figura 8 representa las temperaturas de confort para el aire interior y la temperatura de bulbo seco en el aire exterior mensualmente, como datos de la simulación en DesignBuilder, siendo la diferencia de temperatura de hasta $3{ }^{\circ} \mathrm{C}$ en el mes más cálido.

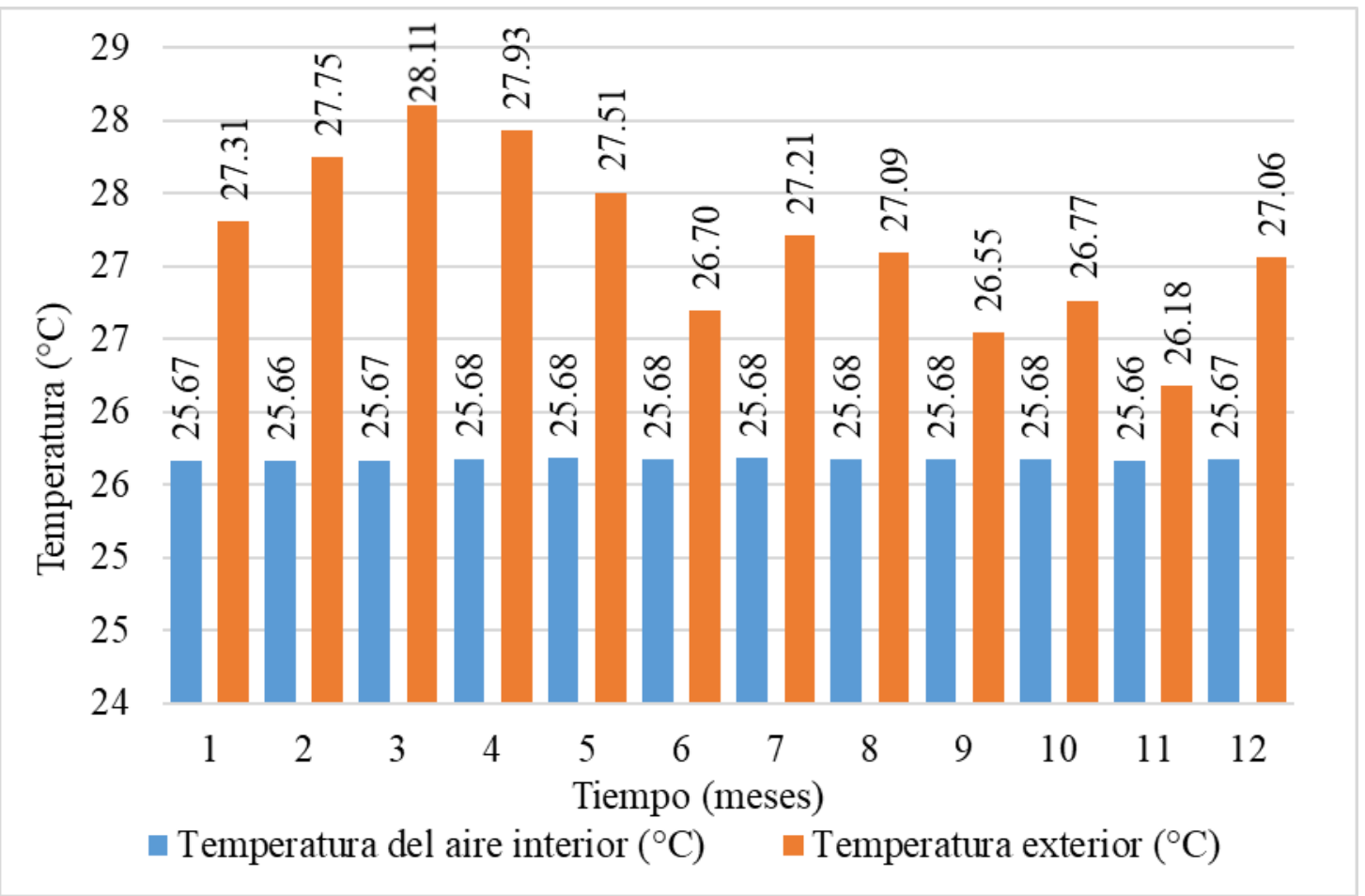

Figura 8: Temperaturas del aire en el interior del recinto y del aire exterior, basado en calendario anual enero diciembre. 


\section{Temperatura del suelo}

De los gráficos mostrados en la figura 9 y figura 10, se observa la alta sensibilidad del sistema a la amplitud térmica a lo largo del año, tomando como punto de inicio el día primero de enero. Se aprecia que a mayor amplitud $(A s)$ se obtiene un mejor rango de operatividad.

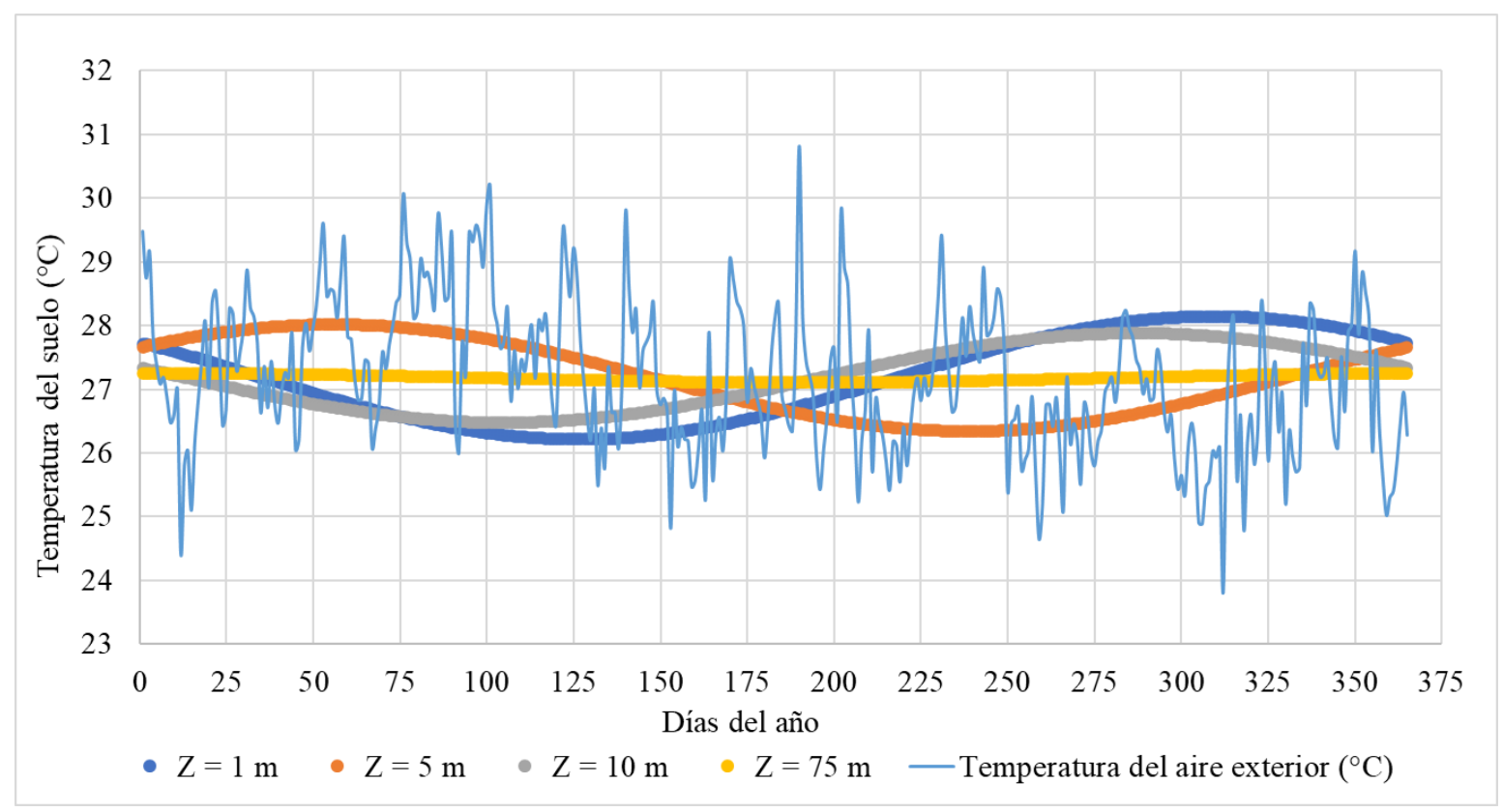

Figura 9: Valores calculados de la temperatura del suelo a diferentes profundidades con una amplitud $A_{S}=1$.

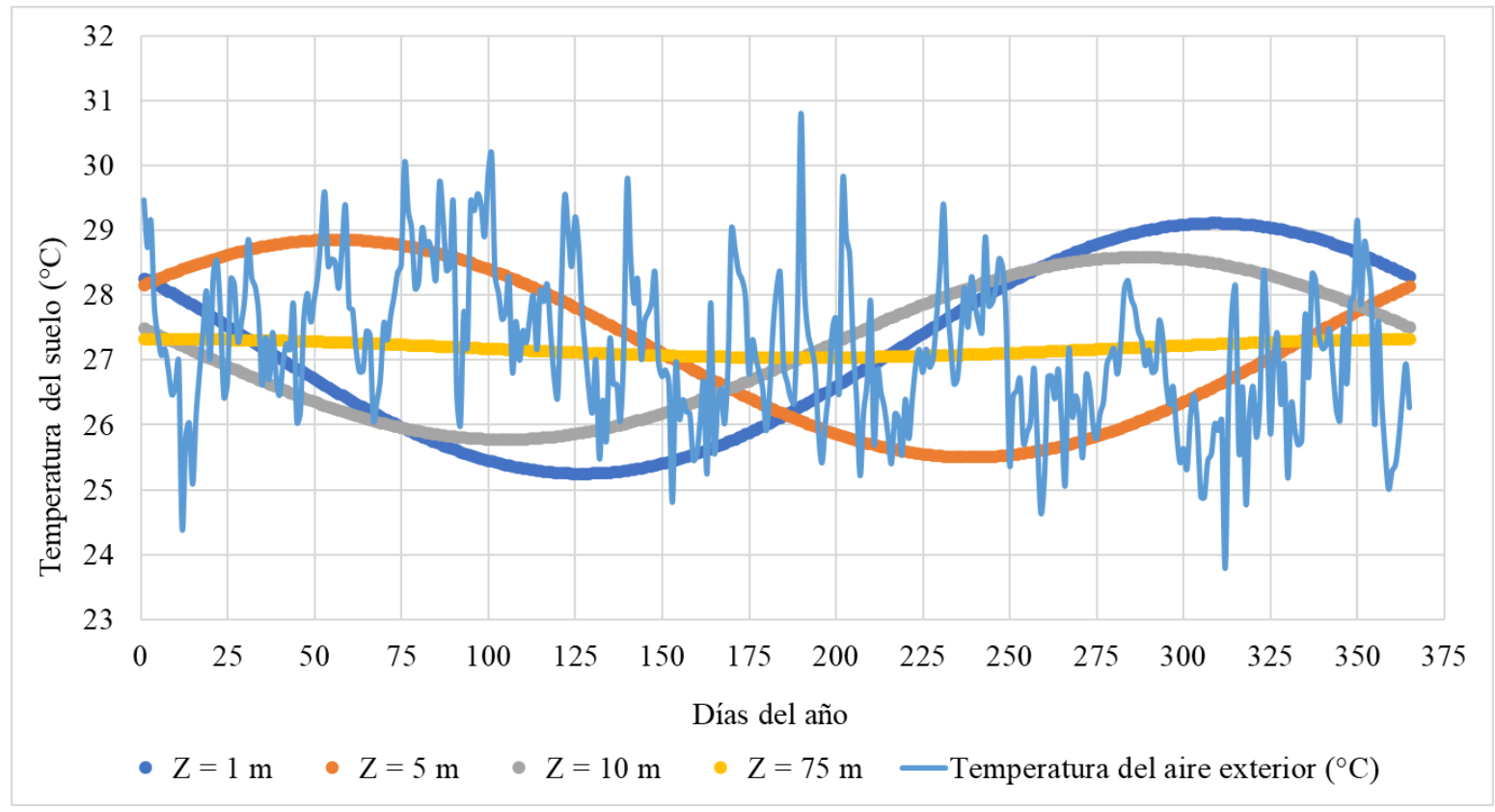

Figura 10: Valores calculados de la temperatura del suelo a diferentes profundidades con una amplitud $A_{S}=2$.

\section{Sistema geotérmico}

El modelo arroja para el caso 1 una longitud total de tubería de $564.68 \mathrm{~m}$, establecidas en un arreglo de 14 tramos de $35 \mathrm{~m}$ horizontal con una separación de $2 \mathrm{~m}$ entre cada tramo y 30 codos tipo estándar a 90 grados para las uniones de los tramos, como lo muestra la figura 11 . 


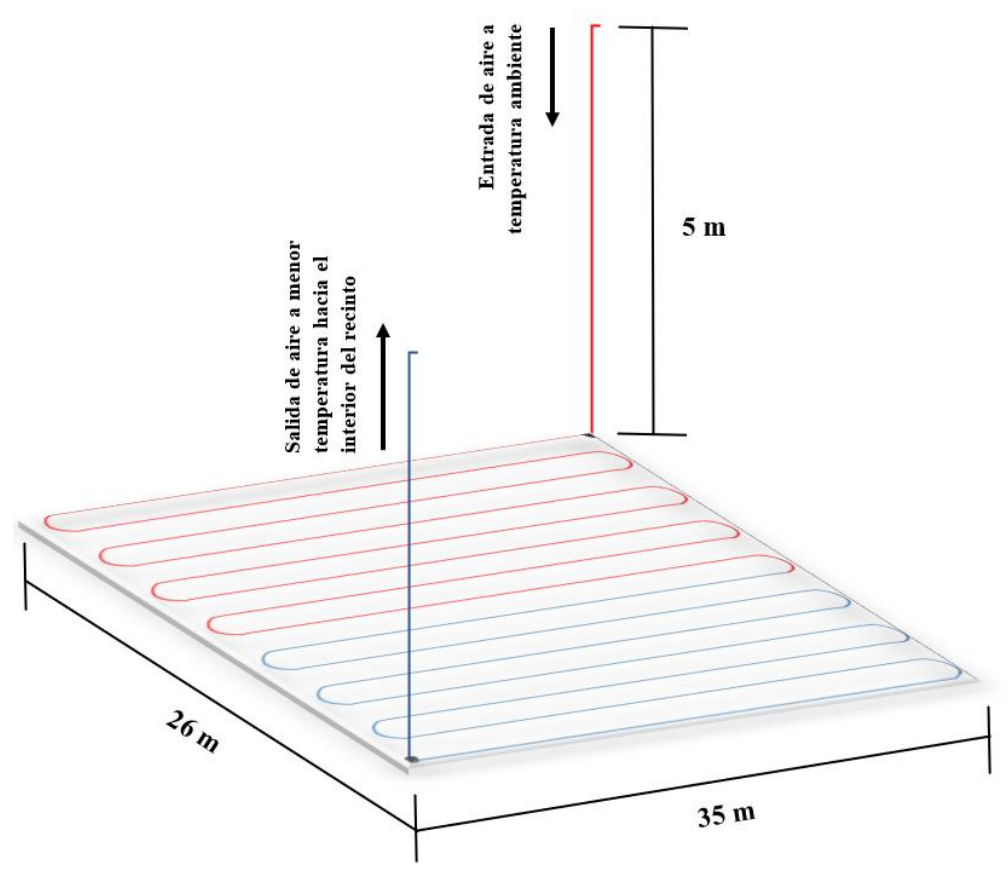

Figura 11: Diagrama representativo del dimensionamiento del intercambiador de calor aire - suelo.

Tomando en consideración esta longitud en conjunto con distintas amplitudes, todas a una profundidad de $5 \mathrm{~m}$ y valores de confort entre $26.1^{\circ} \mathrm{C}$ y $27{ }^{\circ} \mathrm{C}$ (Secretaría Nacional de Energía, 2016), se obtuvo como resultados del caso 2 el gráfico mostrado en la figura 12, en el que la zona verde indica el periodo máximo de operatividad del sistema, y la tabla 5, que muestra la temperatura interior de confort que recibiría la edificación.

Tabla 5: Temperaturas de funcionamiento y días de operación del sistema geotérmico.

\begin{tabular}{cccccc}
\hline Amplitud térmica $(\mathrm{As})$ & 1 & 1.5 & 2 & 2.5 & 3 \\
\hline Temperatura de suelo $\left({ }^{\circ} \mathrm{C}\right)$ & $26.76-26.99$ & $26.34-26.99$ & $25.50-26.99$ & $25.08-26.98$ & $24.66-26.99$ \\
Confort alcanzado $\left({ }^{\circ} \mathrm{C}\right)$ & $26.98-27.09$ & $26.78-27.09$ & $26.38-27.09$ & $26.18-27.08$ & $25.97-27.08$ \\
Operación anual (días) & 130 & 157 & 169 & 171 & 173
\end{tabular}

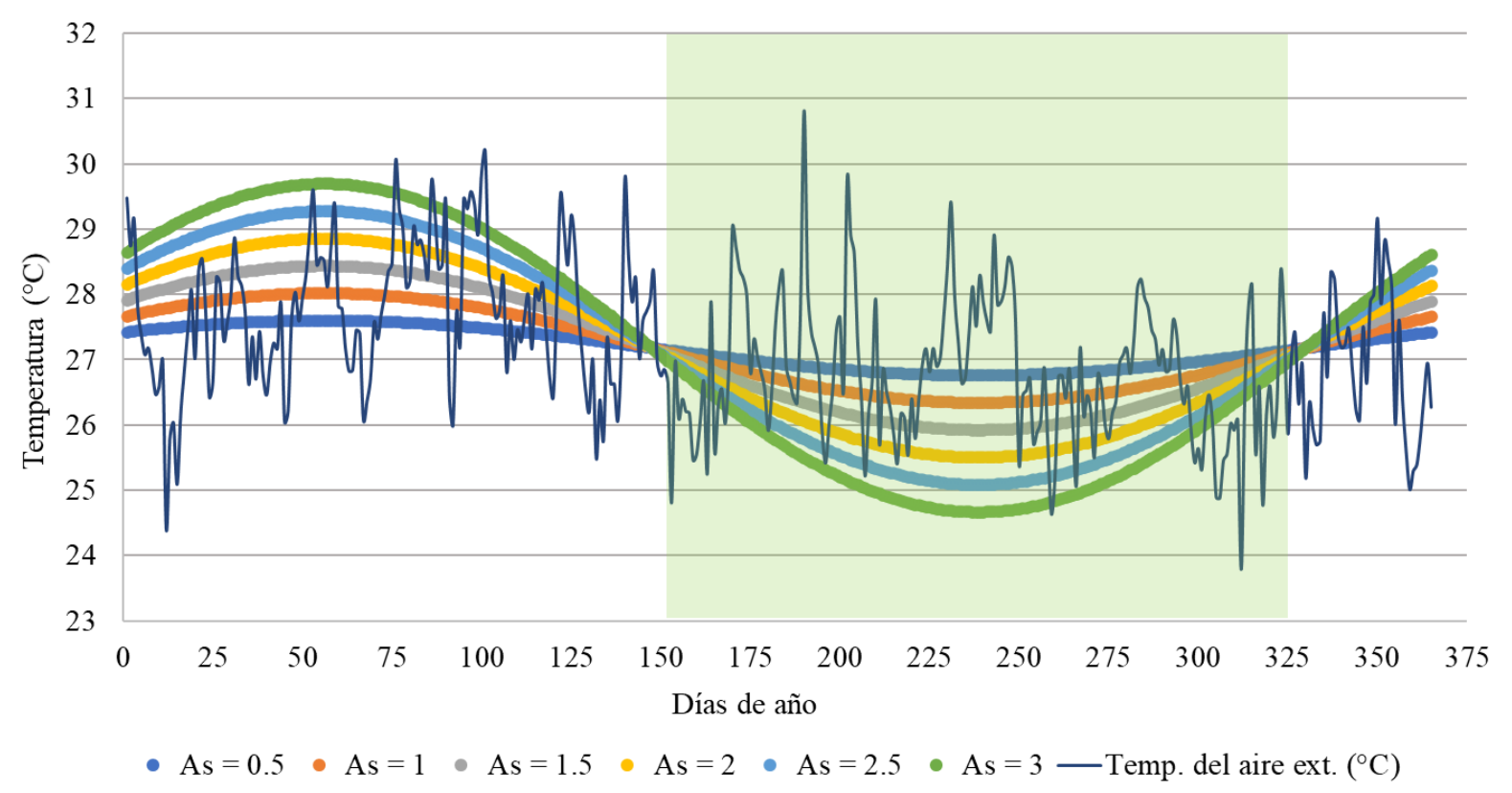

Figura 12: Temperaturas calculadas a $5 \mathrm{~m}$ de profundidad en función de diversos valores de amplitud. 


\section{Generación fotovoltaica}

Para un área total de techo de $1848.33 \mathrm{~m}^{2}$ se estima la utilización de 911 paneles fotovoltaicos del modelo utilizado en (Chen, Chung-Camargo, \& Mora, 2021) lo que resulta en una potencia instalada de $409.7 \mathrm{~kW}$

Tomando en cuenta el ahorro que representa el sistema geotérmico para el consumo eléctrico total, se obtiene que anualmente los paneles solares pueden cubrir hasta 9.29\% de la demanda, como se muestra en la tabla 6.

Tabla 6: Porcentaje de la generación eléctrica mensual y anual cubierta por los paneles fotovoltaicos.

\begin{tabular}{rccc}
\multicolumn{1}{c}{ Mes } & $\begin{array}{c}\text { Potencia generada por } \\
\text { los paneles }(\mathrm{kWh})\end{array}$ & $\begin{array}{c}\text { Consumo total } \\
\text { mensual }(\mathrm{kWh})\end{array}$ & $\begin{array}{c}\text { Razón cubierta por los } \\
\text { paneles }(\%)\end{array}$ \\
\hline Enero & 54472.77 & 686273.42 & 7.94 \\
Febrero & 50666.19 & 618383.27 & 8.19 \\
Marzo & 60219.21 & 685752.81 & 8.78 \\
Abril & 50335.33 & 667646.22 & 7.54 \\
Mayo & 46821.29 & 691161.23 & 6.77 \\
Junio & 48231.78 & 417679.15 & 11.55 \\
Julio & 48552.19 & 431863.60 & 11.24 \\
Agosto & 48694.98 & 431778.85 & 11.28 \\
Septiembre & 46493.92 & 417770.82 & 11.13 \\
Octubre & 48144.72 & 431879.03 & 11.15 \\
Noviembre & 42373.90 & 491854.16 & 8.62 \\
Diciembre & 50345.78 & 688272.40 & 7.31 \\
Anual & 595352.06 & 6660314.96 & 9.29
\end{tabular}

\section{Discusión}

\subsection{Temperatura del suelo}

Dentro de las curvas generadas a partir de la ecuación (2) se encuentra el parámetro de amplitud térmica el cual como se mencionó es medido de forma experimental. Por lo tanto, el enfoque de los resultados obtenidos al no contar con estos datos para la región de estudio fue el de comprobar la sensibilidad del sistema a la amplitud térmica y mostrar el grado necesario para que el sistema geotérmico sea viable de aplicar. De esta manera, los gráficos mostrados en las figuras 9 y 10 reflejan los valores obtenidos de temperatura del suelo a distintas profundidades, reflejando que a medida que aumenta la profundidad, los valores de la temperatura se estabilizan. Esto comprueba lo postulado por Argot en su primera ley: "La amplitud de las oscilaciones de temperatura del suelo disminuye geométricamente cuando la profundidad aumenta aritméticamente", por lo que a una profundidad mayor que sobrepasa la llamada cota isotérmica del suelo, que es la profundidad en la que no se registran variaciones de temperatura, el aumento de la temperatura es bajo, promediado en estudios en $1^{\circ} \mathrm{C}$ por cada $33 \mathrm{~m}$ (Universidad Nacional de La Plata, 2017).

Los gráficos obtenidos muestran que una profundidad de $5 \mathrm{~m}$ es aceptable para iterar las variaciones del sistema geotérmico analizado en el caso 2, dado que lo que se busca son valores de temperatura que permitan un intercambio favorable entre aire y suelo. 


\subsection{Sistema geotérmico}

Para amplitudes bajas se observa que el intercambio de calor es prácticamente nulo por lo que el sistema es factible para amplitudes térmicas mayores de 2.5, dado que no solo sería capaz de funcionar durante la mayor parte del segundo semestre como se muestra en la figura 12 (del día 152 del año que corresponde al primero de junio hasta el día 325, que corresponde al veintiuno de noviembre), sino que a medida que crece la amplitud las variaciones de temperatura son mayores por lo que tendríamos mejores temperaturas de confort en el recinto.

Adicionalmente, considerando los días que el sistema se encargaría de la refrigeración de la edificación dentro de los gastos por enfriamiento actuales de la misma, estos datos derivados de la simulación realizada en DesignBuilder como se muestra en la figura 7, la implementación de geotermia inversa significaría un ahorro del $47.83 \%$ en gastos lo que a su vez representa una disminución en el consumo energético.

Se pueden contrastar estos resultados con los publicados por (Belatrache et al., 2017), donde se reporta una longitud total de tubería de $45 \mathrm{~m}$ la cual es una cifra $90 \%$ más pequeña que la obtenida en el caso 1. Para comprender esta diferencia se analizan primeramente las temperaturas del aire, dado que el estudio de comparación se realizó en un medio árido donde hay meses que registran altas temperaturas de hasta $46^{\circ} \mathrm{C}$ y a su vez temperaturas muy bajas de hasta $3.8{ }^{\circ} \mathrm{C}$, lo que indica fluctuaciones continuas que diferencian las temperaturas aire - suelo en hasta $20.7^{\circ} \mathrm{C}$ en el mes más eficiente, lo que por consiguiente acorta el largo necesario de tubería.

Otro parámetro para considerar es el punto de diseño seleccionado, el informe reporta temperaturas interiores de $33^{\circ} \mathrm{C}$, lo cual no sería conveniente en el caso de Panamá dado que ya no se registrarían temperaturas de confort; al mantener temperaturas interiores más altas se requiere menor longitud de tubería, esto resultado de la alta diferencia de temperaturas, donde la caída de temperatura es muy rápida y tiende a estabilizarse a mayores longitudes, lo que sería poco ventajoso en el caso de comparación.

\subsection{Generación fotovoltaica}

El sistema geotérmico representa un ahorro considerable en refrigeración por lo que facilita una cobertura más amplia del sistema fotovoltaico el cual aprovechando al máximo el área del techo se puede obtener hasta un $11.55 \%$ en los meses que el gasto de refrigeración es menor en cuanto el consumo energético y un ahorro mínimo en de $6.77 \%$ en el mes más caliente o en el periodo que el gasto de refrigeración no es cubierto por el sistema geotérmico (Tabla 7). Este ahorro pudiese incrementarse a futuro disponiendo de un área cercana a la edificación para colocar más paneles fotovoltaicos que contribuyan al sistema. Además, estos datos son comparables con otro estudio realizado en Ciudad de Panamá para las

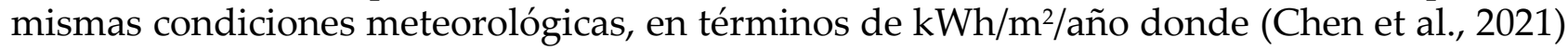
presentan una generación de $214 \mathrm{kWh} / \mathrm{m}^{2} /$ año para una residencia, lo que resulta menor a lo que puede generar la instalación hospitalaria Dr. Carlos N. Brin $\left(322.1 \mathrm{kWh} / \mathrm{m}^{2} / \mathrm{año}\right)$. Es una diferencia aceptable entre ambos valores dada la comparación entre una residencia y un hospital.

\section{Conclusiones}

En la evaluación del potencial del sistema geotérmico inverso, la temperatura del suelo es un parámetro crítico que se debe conocer en detalle, considerando su valor a diferentes profundidades y su variación a lo largo del año en la zona de estudio. Estos últimos detalles 
fueron una limitante en el estudio presentado; sin embargo, el análisis térmico permitió su identificación e importancia. En este sentido, de lograr niveles adecuados en este parámetro crucial, la energía geotérmica puede generar ahorros significativos para edificaciones, en cuanto a la demanda de acondicionamiento de aire; aunque en este trabajo no se consideró, los gráficos muestran oportunidades adicionales en el uso para calefacción y agua caliente sanitaria en algunos meses del año. El análisis económico de implementar dicho sistema tampoco fue abordado en este trabajo.

De igual manera, la generación fotovoltaica estimada, presentó un aporte considerable al consumo eléctrico de la edificación, mostrando su potencial de implementación, considerando que el uso de energía solar en el país ha crecido exponencialmente en los últimos años, en busca de alcanzar las metas energéticas. Vemos que su aplicación en hospitales contribuiría a la reducción de costos en energía; rubro que tiene una tendencia al alza, por lo que todo ahorro puede considerarse importante. Es de suma importancia la actualización de datos referentes a las entidades gubernamentales, como auditorías energéticas y de inmuebles, puesto que para este trabajo se realizaron estimaciones idealizadas respecto al consumo eléctrico y el número de equipos con los que cuenta el hospital al no conocerse estadísticas oficiales de dichas cifras. Finalmente, siendo que en Panamá no existen datos concluyentes sobre el potencial geotérmico, los resultados de esta investigación deben corroborarse con evaluaciones en campo. Por eso este trabajo espera sentar bases para futuras investigaciones que expandirían la matriz energética del país, de considerar este sistema viable.

\section{Contribución de los autores}

En concordancia con la taxonomía establecida internacionalmente para la asignación de créditos a autores de artículos científicos (https://casrai.org/credit/ ). Los autores declaran sus contribuciones en la siguiente matriz:

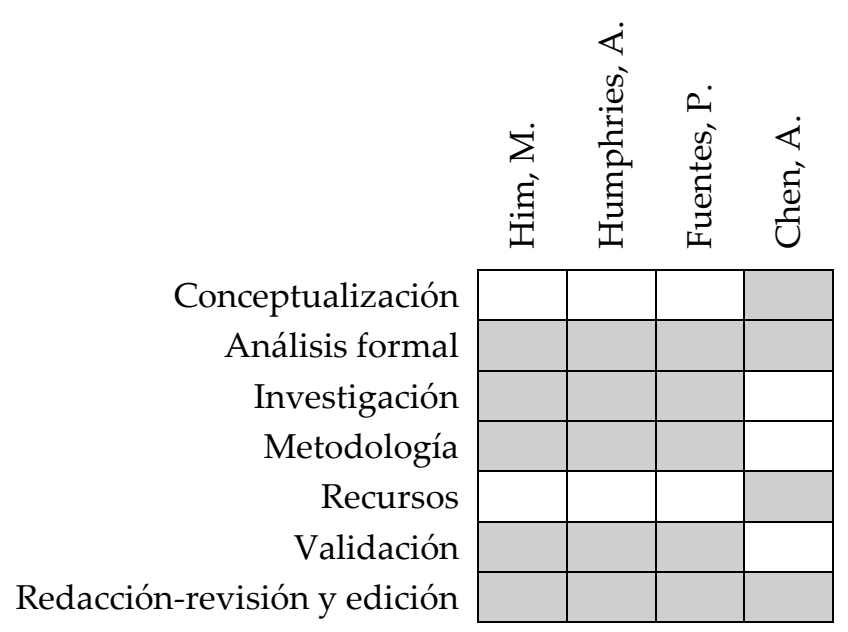

\section{Conflicto de Interés}

Los autores declaran que no existen conflictos de interés de naturaleza alguna con la presente investigación. 


\section{Agradecimientos}

A los arquitectos de la sección DINISA de la Caja de Seguro Social, por su diligencia para facilitar los planos oficiales de la instalación analizada. Los autores agradecen a la Facultad de Ingeniería Mecánica de la Universidad Tecnológica de Panamá por su colaboración. Esta investigación fue financiada por la Institución Panameña secretaria nacional de Ciencia, Tecnología e Innovación (SENACYT) https://www.senacyt.gob.pa/, bajo el proyecto con código FID18-056 y del Sistema Nacional de Investigación (SNI).

\section{Referencias}

Autoridad Nacional de Ambiente. (2011). Atlas Ambiental. Recuperado de: https://www.sinia.gob.pa/index.php/atlas-ambientales

Autoridad Nacional de los Servicios Públicos. (2018). Energía fotovoltaica toma fuerza en interior del país . Recuperado de: https://www.asep.gob.pa/?p=15394

Belatrache, D., Bentouba, S., \& Bourouis, M. (2017). Numerical analysis of earth air heat exchangers at operating conditions in arid climates. International Journal of Hydrogen Energy, 42(13), 8898-8904. https://doi.org/10.1016/J.IJHYDENE.2016.08.221

Cengel, Yunus A.; Ghajar, A. J. (2011). Transferencia de Calor y Masa: Fundamentos y Aplicaciones (4th ed.). México: McGrawHill Educación.

Chen, M., Chung-Camargo, K., \& Mora, D. (2021). Review of Zero Energy Building ConceptDefinition and Developments in Latin America: A Framework Definition for Application in Panama. Energies 2021, Vol. 14, Page 5647, 14(18), 5647. https://doi.org/10.3390/EN14185647

Elvis, I., Soto, P., \& Dixon, J. W. (2005). Celdas fotovoltaicas en egeneración distribuida. Recuperado de: https://hrudnick.sitios.ing.uc.cl/paperspdf/pereda.pdf

Escalante, V., \& Villalba, J. A. (2018). Lineamientos técnicos y económicos para la implementación de un sistema de energía geotérmica de baja entalpía caso de estudio : vivienda unifamiliar en el municipio de Anapoíma-Cundinamarca. Universidad Piloto de Colombia, Colombia. Recuperado de: http://repository.unipiloto.edu.co/handle/20.500.12277/8745

ETESA. (2010). Potencial Geotérmico. Recuperado de:

http://www.etesa.com.pa/estudios.php?act=geotermico

ETESA. (2018). Plan de Expansión del Sistema Interconectado Nacional 2017 - 2031. Panamá.

Google Earth. (2021). Google Earth. Recuperado January 30, 2022, de https://earth.google.com/web/search/Policlinica+Dr.Carlos+N+Brin+(San+Francisco), +Calle+67+1\%2F2+Este,+Panama+City/@8.9972697,-

79.5112718,19.84335462a,1042.00311886d,35y,0h,45t,0r/data=CrkBGo4BEocBCiUwe DhmYWNhOWFhNDhkMTRmZmI6MHg1YTk1YmQzYTRiZGQzODc3GZ88LN

Grupo Ibal. (2019). ¿Qué es la energía fotovoltaica? Recuperado de http://grupoibal.com/fotovoltaica/\#: :text=Es la transformación directa de,una pequeña diferencia de potencial. 
Grupo Visiona. (2013). Tipos de Geotermia - Geotermia. Recuperado de: http://www.grupovisiona.com/es/geotermia/tipos-geotermia

Hernández, F. A., Lemus, I. A., Solano, F. E., \& Martínez, L. A. (2020). ¿Qué características se requieren para que un edificio sea cero energía neta con un costo mínimo de ciclo de vida en la región de Centroamérica y el Caribe? CONIA. Recuperado de http://repositorio.uca.edu.sv/jspui/handle/11674/3804

Jiménez, A., Leal, J. P., \& Garzón, O. L. (2016). Beneficios en la implementación de un sistema de energía solar en hospitales a nivel internacional. AREANDINA. Recuperado de: https://digitk.areandina.edu.co/handle/areandina/1075

Secretaría Nacional de Energía. (2015). Panamá: Programa Nacional de Estadísticas Energéticas. Recuperado de:

https://sustainabledevelopment.un.org/content/documents/13109Panama.pdf

Secretaría Nacional de Energía. (2016). Resolución $N^{\circ}$ 3142. Panamá. Recuperado de: https://www.gacetaoficial.gob.pa/pdfTemp/28165/GacetaNo_28165_20161124.pdf

Teba, C. (2020). NZEB: Qué es y que no es un Edificio de Consumo Casi Nulo I DEXMA. Recuperado de: https://www.dexma.com/es/blog-es/que-son-los-edificios-nzeb-ycomo-contribuyen-el-desarrollo-sostenible/

Tokman, M. (2008). Política Energética. Recuperado January 29, 2022, de Comisión Nacional de Energía. Recuperado de : https://www.cne.cl/archivos_bajar/Politica_Energetica_Nuevos_Lineamientos_08.df

Universidad Nacional de La Plata. (2017). Guía de estudio N³ Aspectos climáticos de la temperatura del suelo. Recuperado de:

https://aulavirtual.agro.unlp.edu.ar/pluginfile.php/34659/mod_resource/content/1/Guia de estudio 3 - 2017- Temperatura del suelo.pdf

Uribe, D. F., \& Arboleda, A. F. (2015). Centros especializados de san vicente fundación: Hospital verde con certificación leed. Revista Ingeniería Biomédica, 9(18), 51-56. https://doi.org/10.14508/rbme.2015.9.18.51-56

Valdés, I. (2018). Abastecimiento energético al hospital de Gorliz mediante energías renovables. (Trabajo de Grado). Bilbao, España: Universidad del País Vasco. Recuperado de: https://addi.ehu.eus/bitstream/handle/10810/29150/TFG Igor Ballesteros Valdés.pdf?sequence $=1 \&$ isAllowed $=\mathrm{y}$

Zomer, C., Urbanetz, J., \& Rüther, R. (2011). On the compromises between form and function in grid-connected building-integrated photovoltaics (BIPV) at low-latitude sites. 30th ISES Biennial Solar World Congress 2011, SWC 2011, 3, 2204-2214. https://doi.org/10.18086/SWC.2011.17.32

Zyvith, Z., Yong, A., Lamantia, M. R., Trevena, M., Lamantia, R., Sharp, L., \& Haghani, S. (2018). Geothermal Heating/Cooling in Massachusetts General Hospital. 2018 ASEE Mid-Atlantic Section Spring Conference. Recuperado de: https://peer.asee.org/29463 